\title{
LITERATICS: UN AMBIENTE VIRTUAL DE APRENDIZAJE DIRECCIONADO A LA SENSIBILIZACIÓN PARA EL ESTUDIO DE LA LENGUA ESPAÑOLA
}

\author{
LITERATICS: UM AMBIENTE VIRTUAL DE APRENDIZAGEM VOLTADO A \\ SENSIBILIZAÇÃO PARA O ESTUDO DA LENGUA ESPANHOLA
}

\section{LITERATICS: A VIRTUAL LEARNING ENVIRONMENT FOCUSED ON SENSITIZATION TO THE STUDY OF SPANISH LANGUAGE}

\author{
${ }^{1}$ Renan Cardozo Gomes da Silva; ${ }^{2}$ Dra. Luciana Contrera Domingo \\ ${ }^{1}$ renancardozo@gmail.com, Universidade Federal do Pampa; ${ }^{2}$ ludomingo@gmail.com, \\ Universidade Federal do Pampa.
}

\begin{abstract}
Resumen
Considerando nuestro contexto de frontera Jaguarão - BR y Río Branco - UY, este trabajo objetiva presentar un ambiente virtual de aprendizaje (AVA) destinado a los alumnos del Proyecto de Enseñanza "LITERATICS: sensibilización para el estudio de la lengua española”. El proyecto tuvo por objetivo sensibilizar los académicos del Curso de Letras de la Universidade Federal do Pampa - UNIPAMPA, Campus Jaguarão, para el estudio de la lengua española a partir de la literatura y de las TIDCs, creando un ambiente de interacción en perspectiva intercultural. El curso tuvo un total de $60 \mathrm{~h}$, divididas en $30 \mathrm{~h}$ presenciales y $30 \mathrm{~h}$ a distancia; hasta el presente momento, ya fueron desarrolladas cuatro ediciones; en la primera usamos diferentes géneros literarios de la latinoamérica, en la segunda usamos el género novela y sus relaciones con los espacios geográficos específicos, en la tercera desenvolvemos actividades a partir de material literario y sus versiones cinematográficas, y en la cuarta utilizamos otra novela que trataba de nuestro cotidiano fronterizo. Sin embargo, en la cuarta edición no utilizamos el AVA. Además, en el ambiente virtual propusimos actividades privilegiando dos destrezas (escrita y lectora), visando la interacción y el proceso de enseñanza-aprendizaje en un ambiente online. Durante el curso fomentamos la búsqueda de informaciones relevantes, visitas a lugares por medio de la red, elaboración de textos, adquisición de léxico y utilización de aplicativos para auxiliar las actividades.
\end{abstract}

Palabras clave: LITERATICS, TDICs, enseñanza-aprendizaje, lengua española.

\section{Resumo}

Considerando o contexto de fronteira Jaguarão - BR e Río Branco - UY, este trabalho objetiva apresentar um ambiente virtual de aprendizagem (AVA) destinado aos alunos do Projeto de Ensino "LITERATICS: sensibilización para el estudio de la lengua española”. O projeto teve por objetivo sensibilizar os acadêmicos do Curso de Letras da Universidade Federal do Pampa - UNIPAMPA, Campus Jaguarão, para o estudo da língua espanhola a partir da literatura e das TIDCs, criando um ambiente de interação a partir de uma perspectiva 
intercultural. O curso teve um total de $60 \mathrm{~h}$, divididas em $30 \mathrm{~h}$ presenciais e $30 \mathrm{~h}$ a distância; até o presente momento, já foram desenvolvidas quatro edições: na primeira usamos diferentes gêneros literários destinados à literatura latino-americana, na segunda usamos o gênero novela e suas relações com os espaços geográficos específicos, na terceira desenvolvemos atividades a partir de material literário e sua versão cinematográfica, e na quarta utilizamos outra novela que tratava do nosso cotidiano fronteiriço. Entretanto, na quarta edição não utilizamos o AVA. No ambiente virtual, propomos atividades visando duas destrezas (escrita e leitura), visando a interação e o processo de ensino-aprendizagem em um ambiente online. Durante o curso, fomentamos a busca de informações relevantes, visitas a lugares por meio da rede, elaboração de textos, aquisição de léxico e utilização de aplicativos para auxiliar as atividades.

Palavras-chave: LITERATICS, TDICs, ensino-aprendizagem, língua espanhola.

\begin{abstract}
Considering our border context (Jaguarão-BR and Rio Branco-UY), this paper aims to make a present of the virtual learning environment (VLE) destined for the students of the teaching project "LITERATICS: sensibilización para el estudio de la lengua española", that aims sensitize the students of the course of Letters of the Federal University of Pampa - UNIPAMPA, campus Jaguarão, for the study of the Spanish language based on an literature and DICTs approach, creating an environment of interaction that aiming an intercultural perspective. The course had a total of 60 hours, split into $30 \mathrm{~h}$ physically and $30 \mathrm{~h}$ at distance. Until now, have been developed four editions: in the first edition have been used different literary genres destined to Latin American literature, in the second we used the novel genre and their relationship to specific geographic spaces, in the third we have developed activities based on literary material and its respective film version, and, finally, in the fourth edition, we used another novel that comes to our border everyday life, however, in this latest edition, we do not used the AVA. In addition, in the virtual environment, we suggest activities aimed to two skills (reading and writing), to the interaction and the process of teaching and learning in an online environment, as well as foster the search for relevant information, visits to places across the network, preparation of texts, vocabulary acquisition and verification of applications to help during the activities.
\end{abstract}

Keywords: LITERATICS, DICTs, teaching and learning, Spanish language.

\title{
1. Introducción
}

Con la emergencia de las medios digitales que forman los diferentes modos de comunicación, la sociedad comienza a agregar en sus paradigmas la integración de los sistemas electrónicos, añadiendo en su rutina las Tecnologías Digitales de la Información y Comunicación (TDICs). Considerando este contexto, este trabajo objetiva presentar un ambiente virtual de aprendizaje (AVA) destinado a los alumnos del Proyecto de Enseñanza LITERATICS: sensibilización para el estudio de la lengua española. El Proyecto tuvo como objetivo sensibilizar los académicos del Curso de Letras de la Universidade Federal do Pampa - UNPAMPA, Campus Jaguarão, para el estudio de la lengua española a partir de la literatura y las TDICs, visando un ambiente de interacción y enseñanza-aprendizaje en una perspectiva intercultural, estableciendo espacios alternativos que posibiliten lograr la adquisición del idioma.

A lo largo de este relato haremos una breve discusión sobre la Era de la Información, en la cual los aparatos electrónicos toman cada vez más espacio en nuestro cotidiano; explicaremos, cómo se desarrolló cada edición del proyecto y la función del AVA durante cada edición, además de reflexionar sobre su papel en el curso. Para realizar tal discusión nos 
anclamos en Castells (2011) en lo que se refiere a los avances sociales y la Era de la Información y Santaella (2004) identificando los diferentes tipos de lectores de la red.

Elegimos trabajar durante el curso con un AVA, pues creíamos que complementaría las actividades, conectando los estudiantes por medio de chats, haciendo que los hábitos de la sociedad actual estén más próximos. Además, en la red están disponibles informaciones que servirán para auxiliar los alumnos, estimulando el educando a conducir su comprensión y distinguir las varias informaciones disponibles.

Así, enfocamos en el uso de las TDICs como medio de auxilio en la enseñanzaaprendizaje de la lengua, teniendo como objetivo específico: discutir a respecto de la Era de la Información, reflexionar sobre el proceso de enseñanza-aprendizaje en una perspectiva intercultural y exponer nuestra visión frente al proyecto.

\section{Era de la Información: interacción y enseñanza-aprendizaje}

Con los constantes avances de la sociedad, las tecnologías digitales comienzan a difundirse rápidamente en las más diversas áreas del conocimiento, siendo incorporadas gradualmente al cotidiano de las personas, al punto de volverse indispensables. La sociedad actual vive la ascensión de las tecnologías y, sobre todo, de internet, que disemina los efectos de la informatización y de la instantaneidad. Según Castells,

O que caracteriza a atual revolução tecnológica não é a centralidade de conhecimentos e informação, mas a aplicação desses conhecimentos e a informação para a geração de conhecimentos e de dispositivos de processamento/comunicação da informação, em um ciclo de realimentação cumulativo entre a inovação e seu uso. (CASTELLS, 2011, p. 50)

Con este cambio social, ahora Era de la Información, estamos diariamente ligados por medio de cables, redes y satélites, que anulan las distancias geográficas y nos dejan más próximos, vía ciberespacio. Este nuevo medio de comunicación crea una interconexión mundial para que la comunicación digital sea instaurada a través de: salas de chats, redes sociales y comunidades virtuales. En estos sistemas interactivos, las computadoras establecen ligaciones con el mundo entero, formando una mediación social entre el sujeto y el ciberespacio.

Con la difusión de lo virtual, surge una nueva forma de cultura que abre espacio para la adquisición de nuevos saberes y para la descubierta de nuevos espacios, la cibercultura, que según Levy (2000, p. 257) es definida como una cultura que deriva de las relaciones entre sociedad sin fronteras que difunden sus relaciones a través de las comunidades interactivas. 
Con esta nueva cultura nuestra sociedad se encuentra obligada a cambiar constantemente, reconstruyéndose diariamente. Conforme Lemos,

\begin{abstract}
Para compreendermos os impactos das novas tecnologias na cultura e na comunicação contemporânea, devemos dirigir nosso olhar para a sociedade enquanto um processo (que se cria) entre as formas e os conteúdos (Simmel). [...] Trata-se, a partir da perspectiva formista simmeliana, de mostrar à dinâmica sociotécnica que se instaura nesse final de século misturando, de forma inusitada, as tecnologias digitais e a socialidade pós-moderna, formando a cibercultura (LEMOS, 2010, p. 81).
\end{abstract}

La cibercultura influencia, sobre todo, los jóvenes que adentran lo virtual, enlazados por íconos, links, blogs, redes sociais, aplicaciones y herramientas. En ese contexto, las tecnologías, que ya fueron vistas como instrumento de alienación e individualismo, se convierten en un ambiente de socialización y convivio. Conforme Lemos (2010), el local donde se accede internet se torna un espacio para almacenar y diseminar información, en el cual agentes inteligentes realizan búsquedas de manera personalizada a fin de encontrar la información, o software, que mejor se encaja con el solicitante.

A partir de eso, vemos que los individuos de la sociedad forman lazos de interacción, creando relaciones de interés y comunidades virtuales. Para Recuero (2001), la importancia de estos lazos se da por las relaciones mantenidas a distancia, pues la computadora, en cuanto mediadora y, internet, como medio de interacción, proporcionan que las personas estén siempre en contacto, anulando las distancias territoriales impuestas. Nuevos espacios de interacción virtual están siendo creados/modificados, aunque algunos aun permanezcan sin o con pocos cambios.

Uno de estos espacios son los ambientes virtuales de aprendizaje (AVA), que posibilitan desenvolver propuestas y actividades en el ciberespacio. Mckimm, Jollie y Cantillon apud Pereira, conceptúan los AVA como:

[...] um conjunto de ferramentas eletrônicas voltadas ao processo ensinoaprendizagem. Os principais componentes incluem sistemas que podem organizar conteúdos, acompanhar atividades e, fornecer ao estudante suporte on-line e comunicação eletrônica (MCKIMM, JOLLIE y CANTILLON apud PEREIRA 2007, p. 6).

Las herramientas deben motivar a los estudiantes, fomentando la interacción entre los profesores y los alumnos. Asumiendo una perspectiva vygotskyana, vemos que el AVA visa primeramente la interacción en el nivel social, para después pasar a la interacción con el entorno. 
En resumen, los AVA disponen de materiales en diferentes formatos como: textos, hipertextos, vídeos, canciones y etc., que fomentan el desarrollo de habilidades y la formación de innúmeras modalidades de enseñanza-aprendizaje, además de promover la interacción, interactividad y facultar la individualidad.

\section{LITERATICS: sensibilización para el estudio de la lengua española}

Considerando el contexto de frontera en que estamos ubicados, Jaguarão - BR y Río Branco - UY, y la baja carga horaria dedicada a la lengua española en el curso de Letras Portugués/Español de la UNIPAMPA, Campus Jaguarão, creamos el Proyecto LITERATICS. A lo largo de las clases del Proyecto, visamos desarrollar todas las competencias por medio de los aspectos culturales que esta región ofrece, integrando la aproximación del idioma a las cuestiones de diversidad e interculturalidad típicas y formadoras del contexto híbrido de la frontera.

El Proyecto tuvo un total de $60 \mathrm{~h}$, divididos en $30 \mathrm{~h}$ presenciales y $30 \mathrm{~h}$ a distancia. Hasta el momento fueron realizadas cuatro ediciones, pero solamente en las tres primeras utilizamos el AVA. En la primera edición trabajamos con diferentes géneros literarios destinados a la literatura latinoamericana; en la segunda, titulada "Viajero", usamos una novela infantojuvenil y la tercera, denominada "Otro rollo", desarrollamos actividades de lengua española a partir de material literario y su versión cinematográfica. En las actividades presenciales hicimos la lectura de cuentos, escuchamos músicas, asistimos videos y películas, todo con el objetivo de motivar, estudiar e interactuar en lengua española, promoviendo un ambiente de inmersión.

Durante todas las ediciones trabajamos en una perspectiva intercultural, reconociendo por medio de la literatura diferentes culturas y promoviendo un diálogo entre diferentes grupos socioculturales. Siguiendo los postulados de Candau,

A perspectiva intercultural quer promover uma educação para o reconhecimento do outro, o diálogo entre os diferentes grupos socioculturais. Uma educação para a negociação cultural, o que supõe exercitar o que Santos denomina hermenêutica diatópica. A perspectiva intercultural está orientada à construção de uma sociedade democrática, plural, humana, que articule políticas de igualdade com políticas de identidade (CANDAU, 2008, p.54). 
Siendo así, el sujeto intercultural es aquel que reconoce una o más culturas, estableciendo relaciones entre su cultura y culturas diferentes, sabiendo explicar sus diferencias, además de aceptarlas y valorizarlas (KRAVISKI e BERGMANN, 2006).

Referente al AVA, creado para desarrollar la parte a distancia del Proyecto, fue compuesto por de un muro de noticias donde los alumnos realizaban comentarios, acompañaban las actualizaciones hechas por el administrador, leían noticias de periódicos de Argentina y Uruguay vía Twitter; accedían a un espacio para enviar mensajes con consultas, dudas y sugerencias, a un área para discusión de los participantes a través de foros y a un lugar para compartir todos los materiales utilizados. El acceso se realizaba mediante una cuenta/ perfil personal.

Con el transcurso de cada semana subíamos actividades en los foros y todos los materiales utilizados a lo largo del curso. A cada edición, siempre hacíamos la presentación del Proyecto y explicábamos que parte de la carga horaria, 30h, estaban destinadas a la realización de actividades desarrolladas en el ambiente virtual. Además, al principio de las actividades presenciales ofrecimos un taller visando auxiliar el manejo. En ese momento divulgamos un tutorial para auxiliar las inscripciones.

Creamos el AVA en el sitio FreeWebs, que ofrece diversos layouts y recursos para crear sitios. Elegimos el estilo red social, donde cada alumnos tendría que crear una cuenta, un perfil, subir una foto y, sobretodo, interactuar. A partir de eso, elegíamos un tema de acuerdo a la temática de cada edición. Abajo, presentamos la página inicial de cada edición:

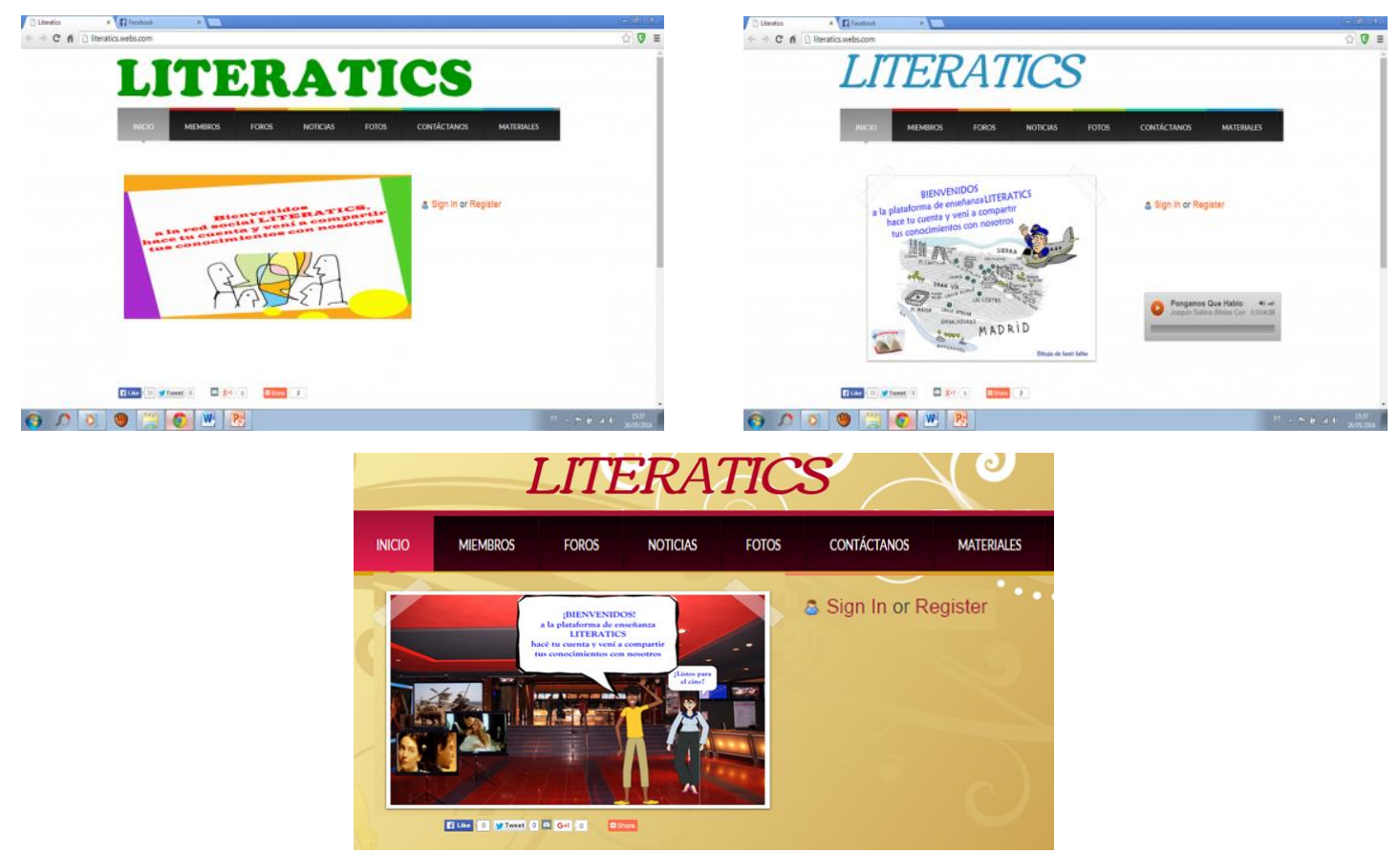


Figura - Layout de la página inicial del proyecto

Fuente: www.literatics.webs.com

\section{Reflexión}

Con el desarrollo de las actividades creemos que los estudiantes tenían facilidad en acceder al AVA, visto que son sujetos oriundos de la Era de la Información, entretanto, la mayoría se mostró con dificultad en desarrollar tales competencias, haciendonos reflexionar sobre el verdadero desempeño de los jóvenes en ambientes virtuales. Conforme Santaella (2004), las dificultades de acceso a internet se dan en todas las fajas etarias, es un mito decir que los jóvenes no poseen dificultades.

Cuando elaboramos el AVA, pensábamos que trabajaríamos, conforme define Santaella (2004), con lectores inmersos o sumergidos, sujetos ligados al ciberespacio que se caracterizan por el uso frecuente de las telecomunicaciones y de las TDICs a fin de obtener informaciones, que son actualizadas cada segundo. Ese lector de la pantalla, conforme la autora, conserva características de los otros dos lectores por ella delimitados. Entretanto, es un lector que "navega em uma tela, programando leituras, num universo de signos evanescentes e eternamente disponíveis, contanto que não se perca a rota que leva a eles" (SANTAELLA, 2004, p. 33); además,

Trata-se, na verdade, de um leitor implodido cuja hipersubjetividade de infinitos textos num grande caleidoscópio tridimencional onde cada novo nó e nexo pode conter uma outra grande rede numa outra dimensão. [...] um universo novo que parece realizar o sonho ou alucinação borgiana da biblioteca de Babel, um biblioteca virtual, mas que funciona como promessa eterna de se tornar real a cada "clique" do mouse. (SANTAELLA, 2004, p.33)

Con el paso del tiempo y el desarrollo de la propuesta, notamos que algunos alumnos no realizaban las actividades y que presentaban dificultad en subir las tareas. Con eso, percibimos que pertenecer a la Era da Información no implica que todos sepamos navegar en el ciberespacio, razón por la cual es un error afirmar que todos dominan las tecnologías digitales. Por eso, Santaella (2004) define usuarios del ciberespacio como los novatos, aquellos que no poseen ninguna familiaridad con el ciberespacio, presentando dificultades en las operaciones durante la navegación; los legos que son los que poseen cierto conocimiento respecto del ciberespacio, saben navegar, pero se limitan a los mismos sitios; los expertos que manipulan las herramientas y los comandos con velocidad y familiaridad. 
Al discurrir de todas las ediciones nos deparamos con los tres usuarios, y fuimos manejando estrategias para auxiliarlos a cada uno. Al final de cada edición, realizamos un cuestionario para conocer la opinión de los alumnos sobre las actividades y, sobre todo, sobre la plataforma. Infelizmente no todos contestaron las preguntas, pero los que lo hicieron, afirmaron no tener problemas en el AVA y que mejorarían algunas cuestiones referentes al sistema, que por veces salía del aire requiriendo actualizaciones constantes. Todos afirmaron estar preparados para utilizar las tecnologías digitales en clase y que la mayoría de las veces que las habían utilizado en clase era para investigar y preparar o presentar trabajos.

Referente a la enseñanza y aprendizaje de la lengua española, todos afirmaron que el Proyecto los ayudó en la pronunciación, adquisición de léxico y conocimiento de la lengua y las diferentes culturas que la componen.

\section{Conclusión}

El Proyecto de Enseñanza LITERATICS: sensibilización para el estudio de la lengua española. curso fue desarrollado durante dos años, destinado en su primera edición solamente para alumnos del primer semestre y las demás para todos los alumnos de Letras, abriendo excepciones para estudiantes de otros Cursos.

Con el término del Proyecto recibimos noticias de progresos de los académicos en las asignaturas de lengua española, sobre todo, en lo que se refiere a la pronunciación y a la escritura. La dinámica de dividir el Proyecto en las modalidades presencial y a distancia, hizo que los alumnos se aproximasen de diferentes culturas, por medio de la lectura, además de ejercitar el uso de diferentes aplicativos, seleccionar informaciones relevantes en la red y realizar visitas virtuales a diferentes lugares, por ejemplo, museos virtuales.

Creemos que el Proyecto los auxilió en todos los componentes curriculares, además de promover el uso de las TDICs en clase. Tuvimos algunos problemas con la utilización del AVA, entretanto, creemos que su uso sirvió para sacar el estudiante de su zona de comodidad, el Facebook, por ejemplo, y hacerlos utilizar otros recursos que poseen la misma dinámica, pero en un formato diferente. Por fin, estamos seguros que el Proyecto tuvo un resultado positivo en lo que se refiere a la cualificación del proceso de enseñanza-aprendizaje, enriqueciendo la formación de los estudiantes. 


\section{Referencias}

BERGMANN, Juliana; KRAVISKI, Elys Regina. Interculturalidade e Motivação na Aprendizagem de Línguas Estrangeiras. Intersaberes (Facinter). Curitiba, v.1, p. 78-86. Disponible en: < http://uninter.com/intersaberes/index.php/revista/article/download/88/62>. Acceso en: 10/11/2015.

CANDAU, Vera Maria Ferrão. Direitos humanos, educação e interculturalidade: as tensões entre igualdade e diferença. Revista Brasil Educacional [online]. 2008, vol.13, n.37, p. 4556. Disponible en: 〈http://www.scielo.br/pdf/rbedu/v13n37/05>. Acceso en: 10/11/2015.

CASTELLS, Manuel. A sociedade em rede. 6.ed. São Paulo: Paz e Terra, 2011.

KERCKHOVE, Derrick de. A pele da cultura. São Paulo: AnnaBlume, 2009.

LEMOS, André de. Cibercultura: tecnologia e vida social na cultura contemporânea. 5.ed. Porto Alegre: Sulina, 2010.

LÉVY, Pierre. Cibercultura. 3.ed. São Paulo: Editora 34, 2000.

PEREIRA, Alice Cybis; SCHMITT, Valdenise; DIAS, Maria Regina Alvares. Ambientes Virtuais de Aprendizagem. In: PEREIRA, Alice T. Cybis. (orgs). AVA - Ambientes Virtuais de Aprendizagem em Diferentes Contextos. Rio de Janeiro: Editora Ciência Moderna Ltda., 2007.

RECUERO, Raquel. Redes sociais na internet. 2.ed. Porto Alegre: Sulina, 2011.

SANTAELLA, Lúcia. Navegar no ciberespaço: o perfil do leitor imersivo. São Paulo: Paullus, 2004.

VIGOSTKY, Lev S. A Formação Social da Mente. 7.ed. São Paulo: Martins Fontes, 2007. 